\title{
Molecular and cellular mechanisms of spastin in neural development and disease (Review)
}

\author{
QIULING LIU, GUOWEI ZHANG, ZHISHENG JI and HONGSHENG LIN
}

\begin{abstract}
Department of Orthopedics, The First Affiliated Hospital of Jinan University, Guangzhou, Guangdong 510630, P.R. China
\end{abstract}
Received July 20, 2021; Accepted September 29, 2021

DOI: $10.3892 / \mathrm{ijmm} .2021 .5051$

\begin{abstract}
Spastin is a microtubule (MT)-severing enzyme identified from mutations of hereditary spastic paraplegia in 1999 and extensive studies indicate its vital role in various cellular activities. In the past two decades, efforts have been made to understand the underlying molecular mechanisms of how spastin is linked to neural development and disease. Recent studies on spastin have unraveled the mechanistic processes of its MT-severing activity and revealed that spastin acts as an MT amplifier to mediate its remodeling, thus providing valuable insight into the molecular roles of spastin under physiological conditions. In addition, recent research has revealed multiple novel molecular mechanisms of spastin in cellular biological pathways, including endoplasmic reticulum shaping, calcium trafficking, fatty acid trafficking, as well as endosomal fission and trafficking. These processes are closely involved in axonal and dendritic development and maintenance. The current review presents recent biological advances regarding the molecular mechanisms of spastin at
\end{abstract}

Correspondence to: Dr Zhisheng Ji or Professor Hongsheng Lin, Department of Orthopedics, The First Affiliated Hospital of Jinan University, 613 Huangpu Avenue, Guangzhou, Guangdong 510630, P.R. China

E-mail: jizhisheng0521@163.com

E-mail: tlinhsh@jnu.edu.cn

Abbreviations: HSP, hereditary spastic paraplegia; ER, endoplasmic reticulum; LD, lipid droplet; HD, hydrophobic domain; MT, microtubule; MIT, MT-interacting and trafficking domain; MTBD, MT-binding domain; AAA, ATPases associated with diverse cellular activities; ESCRT, endosomal sorting complexes required for transport; NES, nuclear export signal; NLS, nuclear localization signal; ATP, adenosine triphosphate; PDZD8, PDZ domain-containing protein 8; SOCE, store-operated calcium entry; MAP, MT-associated protein; KIF5, kinesin-related protein 5; MCS, membrane contact site; NSC, neural stem cell; GRIP, glutamate receptors interacting protein; AMPAR, $\alpha$-amino-3-hydroxy-5-methy 1-4-isoxazolepropionic acid receptor; CK2, casein kinase 2

Key words: spastin, hereditary spastic paraplegia, microtubule, cellular function, neuronal development, HSP-SPG4 the cellular level and provides insight into how it affects neural development and disease.

\section{Contents}

1. Introduction

2. Structure and functions of spastin

3. Regulation of spastin's stability and activity

4. Role of spastin in neural development

5. Role of spastin in HSP

6. Concluding remarks

\section{Introduction}

Spastin, encoded by the SPG4 gene, was identified from mutations in patients with hereditary spastic paraplegia (HSP). HSPs are characterized by progressive lower limb spasticity and weakness. Spastin mutation is the chief cause of HSP and is inherited as an autosomal dominant trait, accounting for $60 \%$ of autosomal dominant cases and $15 \%$ of sporadic cases $(1,2)$. HSP pathogenesis is associated with the degradation of the distal ends of corticospinal axons, which are the major central nervous system pathways connecting the motor cerebral cortex to the spinal cord $(3,4)$. However, the underlying molecular mechanisms of spastin in neural development and HSP have remained to be fully clarified. Recently, numerous spastin-related studies have been performed to identify its cellular roles and elucidate how it affects axonal behaviors, thereby aiding in the development of therapeutic strategies against HSPs.

Spastin isoforms add complexity to the understanding of the pathology and etiology of HSP-SPG4. Spastin has two major isoforms, M1 and M87, attributed to different initiation codons. An additional two spastin isoforms are caused by alternative mRNA splicing of exon $4(4,5)$. Physiologically, M1 is mainly distributed in the adult spinal cord, where the corticospinal axons degenerate in patients with HSP. However, M87 is more abundant and ubiquitously expressed than M1 $(6,7)$. Genetic analyses have revealed that both M1 and M87 are involved in HSP pathology (8-10). With the exclusive hairloop domain, M1 spastin particularly localizes to the endoplasmic reticulum (ER) membrane and lipid droplets (LDs), thus regulating serious cellular activity involving the ER or LDs (11). To date, numerous studies have revealed 
the cellular function of M1, including ER shaping (12), LD metabolism $(11,13)$, membrane remodeling $(14,15)$, endosomal fission (16-18) and fast axonal transport $(19,20)$. However, how M87 works and coordinates with M1 remains largely elusive. Recent studies further highlight the important roles of spastin during neurogenesis (21), axonal development (22-24), synapse formation and spine maturation (25-27). Furthermore, an innovative severing model has been proposed, which provides novel insight regarding microtubule (MT)-related activities, thus reasonably elucidating the mechanisms of the roles of spastin in physiological and pathological conditions $(28,29)$. The present review focuses on recent biological advances and provides insight into the cellular mechanism of spastin and its role in neural development and disease.

\section{Structure and functions of spastin}

Domain architecture and structure of spastin. Spastin is a highly conserved protein whose complex functions are attributed to its multiple modular domain structure and different isoforms. Full-length spastin consists of four functional domains: The hydrophobic domain (HD) situated between residues $1-87$, the MT-interacting and trafficking domain (MIT) spinning (residues 116-194), the MT-binding domain (MTBD) situated in residues 270-328 and the adenosine triphosphatases associated with diverse cellular activities (AAA) domain spinning (residues 342-599). The HD has a high affinity for the membrane structure and determines the subcellular distribution of full-length spastin. MIT is essential for spastin's interaction with endosomal sorting complexes required for transport (ESCRT)-III proteins responsible for cytokinesis completion, endosomal trafficking and other related cellular processes. Similarly, MTBD mediates spastin's interaction with tubulin, which is a prerequisite for severing. By contrast, the AAA domain mediates hexamer formation and catalyzes the severing of MTs $(3,30,31)$.

M1 and M87 are localized in different tissues and subcellular locations. M1 is largely distributed in the cytosol due to the strong nuclear export signal (NES) sequence of 50-87, while M87 is distributed in the whole cell. Spastin also has a nuclear export signal sequence situated in residues 195-204 that spans the MIT and exon 4 region and a nuclear localization signal (NLS) sequence $(32,33)$. Fig. 1 presents the spastin domain architecture and highlights the NES and NLS locations. Although spastin is found in the nucleus, its function in the nucleus remains elusive. A recent study postulated that extracellular histone $2 \mathrm{~B}$ is able to recruit spastin at the midbody during the late stages of abscission (34). By using proteomics analysis, our group also identified the interaction between spastin and numerous histone isoforms (not published). Since histones are vital for the morphology of chromosomes, it may be inferred that spastin is involved in the consolidation of chromosomes or in the regulation of protein transcription. However, this assumption remains to be experimentally validated.

Severing model of spastin. Studies that determined that spastin's structure binds with peptides have revealed its severing mechanism. Spastin forms hexamers through its AAA region in the presence of ATP. The hexamer structure has a central spiral ring structure that is positively charged. The loop subsequently engages with the negatively charged C-termini of tubulin, thereby mechanically extracting tubulin from the lattice using the energy provided by ATP hydrolysis (35-37). Mechanistically, spastin forms open spirals, as the subunits are mutated in an ATP-binding manner. The hexamer moves up to form a closed structure when the spastin structure with adenosine diphosphate-beryllium fluoride represents a 'broken spiral' in which the sixth AAA is in the apo state (36).

Questions to be addressed are whether MT severase is able to depolymerize or amplify MTs, and how severase controls MT length and mass. Recent studies provide answers to these questions and postulate that spastin acts as an amplifier of total MT length and mass $(29,38)$. However, spastin's potential to induce MT regrowth or catastrophe (i.e. the conversion of a growing microtubule into a shrinking one depends on the MT concentration $(29,39,40)$. To date, two major theories for $\mathrm{MT}$ rescue after severing action by spastin have been postulated. According to the first theory, spastin grips the C-terminus of tubulin in an adenosine triphosphate (ATP) hydrolysis manner, followed by refilling the damaged lattice with guanosine triphosphate-tubulin. The MTs severed by spastin are then stabilized and act as seeds to mediate MT regrowth (38). In the second theory, spastin concentrates at the newly generated plus end of MTs to block depolymerizing activity, similar to other MT-associated proteins (MAPs), such as calmodulin regulated spectrin associated protein family member 3. The key evidence for this theory is that the stimulation of increasing the MT length and mass is independent of ATP hydrolysis (29). In general, both theories indicate that spastin is a dual-function enzyme that promotes MT regrowth or catastrophe and provide novel insight regarding its severing activity during physiological conditions.

Over the past decades, spastin was thought to cut long MTs into short MTs to regulate MT dynamics (3). A question may be put forward regarding how MTs behave after severing and how severed MTs contribute to cellular functions. The observations discussed above provide an intuitionistic model and form a foundation to understand MT behavior after severing and provide comprehensive insight into numerous molecular and cellular mechanisms of spastin. The cellular functions of spastin introduced below are based on this model.

Cellular functions of spastin based on its domain interactions Spastin is an MT-severing enzyme containing MTBD and AAA domains with adequate severing activity. In addition, the HD and MIT domains interact with various proteins and recruit spastin to specific subcellular localizations to sever the spatial MTs. Understanding the domain-based interactions may provide insight into spastin-severing activities at the cellular level. The cellular functions of spastin based on its domain interactions are described below and illustrated in Fig. 2.

HD domain wedged into the membrane structure. ER shaping and $\mathrm{Ca}^{2+}$ handling. M1 spastin has an HD region that forms a hairpin loop that embeds into the membrane lipid bilayer, causing spastin to be wedged into the membrane. Three other HSP-related proteins, receptor expression enhancing proteins (REEPs), alastins and reticulons, also have a similar hairpin 


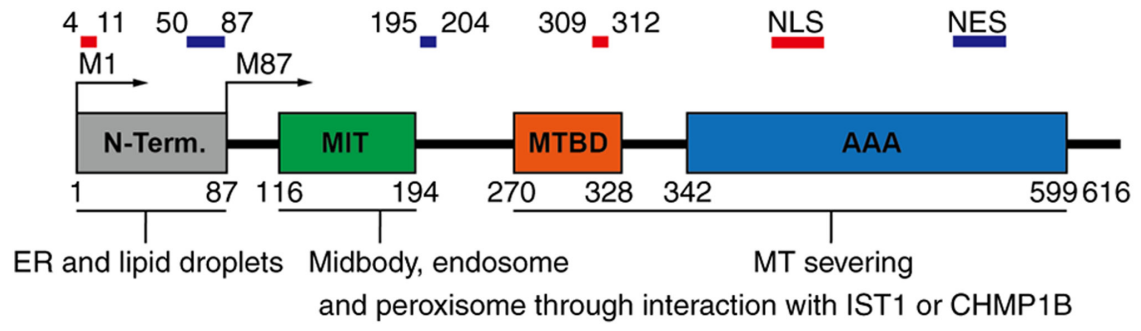

Figure 1. Spastin domain architecture. The domains recruit spastin to specific subcellular localizations to mediate MT severing. The N-terminal domain is responsible for wedging into the ER and lipid droplet membrane. The ESCRT-III proteins IST1 and CHMP1B interact with the MIT domain and recruit spastin to ESCRT-III regions, such as midbody, endosome and peroxisome. MTBD is required for MT binding, while the AAA domain is necessary for MT severing. Two nuclear localization signals (amino acids 4-11 and 309-312) and two nuclear export signals (amino acids 50-87 and 195-204) are responsible for protein shuttling between the nucleus and cytoplasm. MT, microtubule; ER, endoplasmic reticulum; MIT domain, MT interacting and trafficking domain; ESCRT, endosomal sorting complexes required for transport; NES, nuclear export signal; NLS, nuclear localization signal; MTBD, MT-binding domain; AAA, adenosine triphosphatases associated with diverse cellular activities; IST1, increased sodium tolerance 1; CHMP1B, charged multivesicular body protein 1B.

A

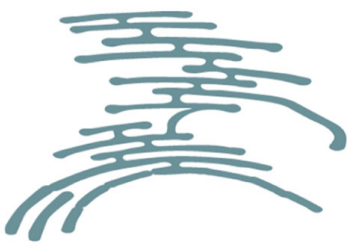

B

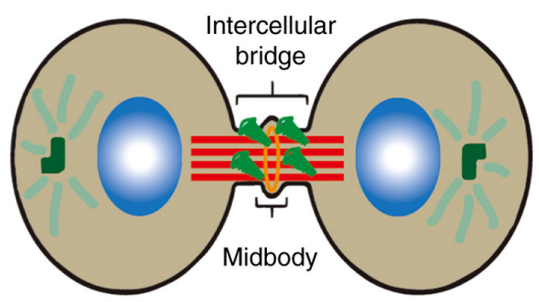

Trans-Golgi
LD-peroxisome

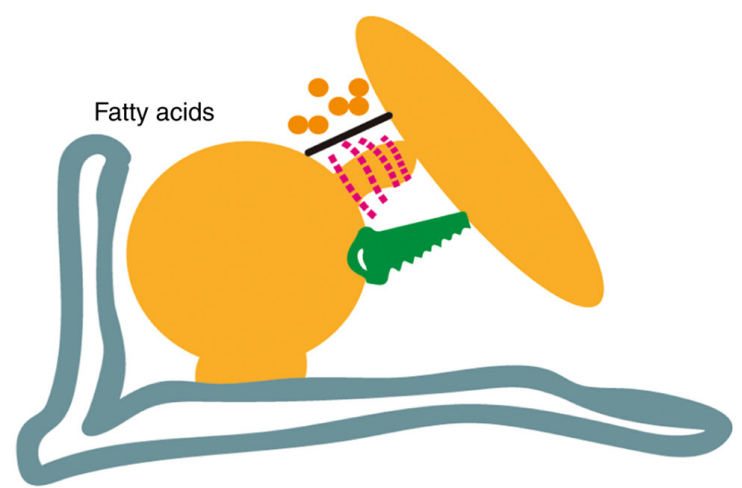

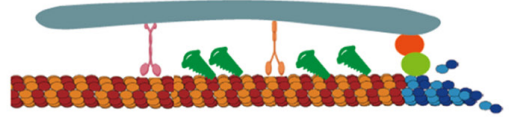

C

Endosome fission and pathway

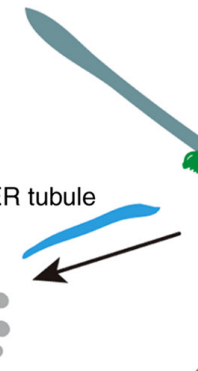

Sorting endosome

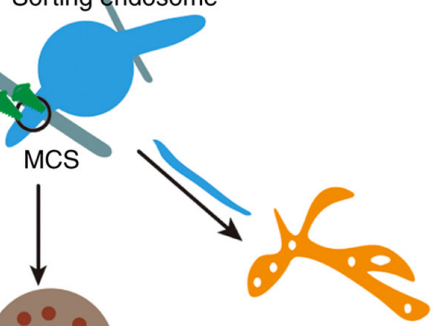

Recycling endosome

Late endosome

$\mathrm{E}$

Endosomal trafficking
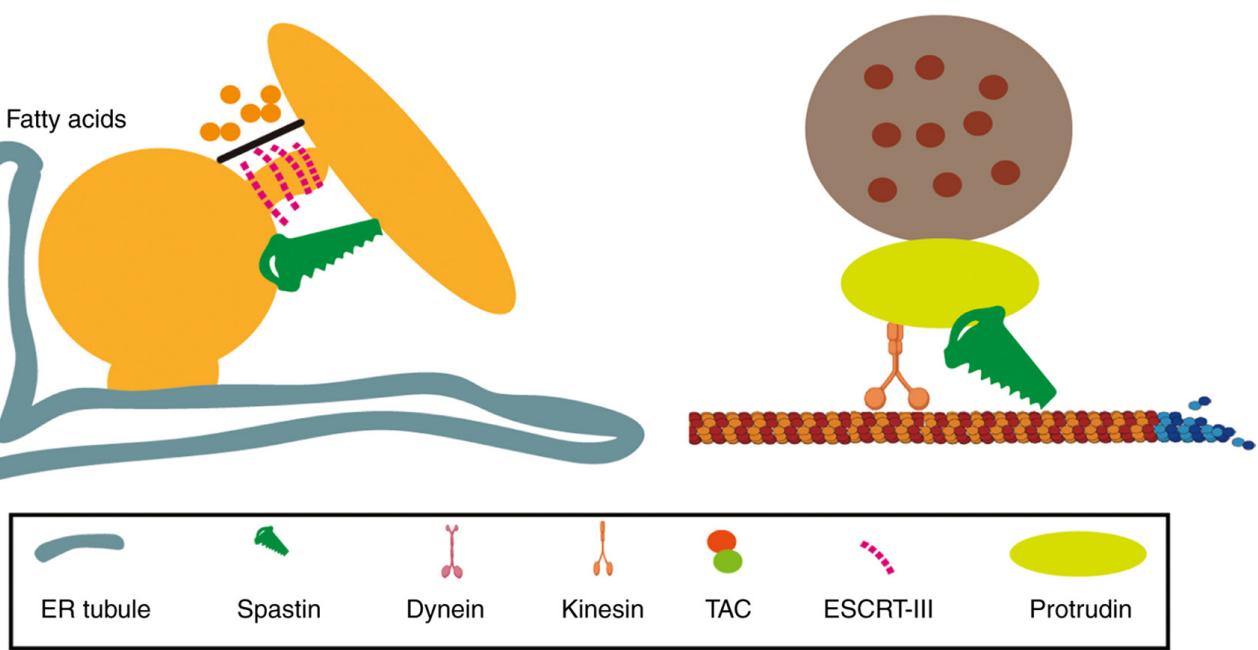

Figure 2. Involvement of cellular activities mediated by severing activities of spastin. Spastin regulates MT-mediated cargo transport and numerous cellular activities associated with MT dynamics. (A) Spastin regulates ER shaping by altering the ER surrounded MT dynamics. MT is linked with ER through the MT plus-end tip attachment complex. (B) Spastin mediates MT clearing in the midbody at the late stage of cytokinesis. (C) Spastin promotes ER tubule and endosome MCS formation and contributes to endosome fission and trafficking through trans-Golgi or recycling pathways. (D) M1 spastin tethers lipid droplets to peroxisomes and directs fatty acid trafficking through ESCRT-III. (E) Spastin negatively regulates late endosome trafficking through interaction with protrudin by breaking the 'railways' of kinesin-mediated transportation. MT, microtubule; MCS, membrane contact site; LD, lipid droplet; ER, endoplasmic reticulum; ESCRT, endosomal sorting complexes required for transport. 
loop and are also recruited in the ER. Thus, these proteins shape the tubular ER $(12,41)$.

Spastin shapes the ER network and affects $\mathrm{Ca}^{2+}$ handling (42). However, the mechanisms of spastin in ER shaping remain to be explored, although numerous HSP-related proteins involved in ER shaping have been largely investigated. The ER sheets in Drosophila melanogaster neurons are larger and have much fewer tubules when the dominant-negative variant of the MT-severing protein spastin is used to induce MT stabilization. Furthermore, the store-operated calcium entry (SOCE) process is inhibited, affecting the flies' flight ability. However, promoting MT dynamics by applying the MT-destabilizing drug vinblastine restores flies' ER morphology, SOCE and flight ability (42). These results suggest that the MT dynamics provided by spastin have a decisive role in forming ER tubules. An important question is whether the severing activity mediated by spastin affects ER morphology. Currently, there is no direct evidence of how spastin affects MTs surrounding the ER. As discussed above, MT dynamics have an important role in ER morphogenesis. There are two conditions under which MTs direct the fate of ER morphology: i) Kinesin-MT-mediated transport drives ER tubules; and ii) the ER tubules move along the growing MT by docking onto the plus-end through a tip attachment complex (43-45). Combined with the innovative hypothesis of the severing model, it is reasonable that spastin promotes ER tubule formation by increasing MT fragments with more MT-plus ends (38). Furthermore, the increase in the total length of the MT potentially leads to more kinesin loading, resulting in more efficient transport.

Another potential mechanism of spastin-mediated ER shaping is through interactions with protrudin. Protrudin is also an ER-resident protein and regulates the sheet-to-tubule balance $(46,47)$. Kinesin-related protein 5 (KIF5) belongs to the kinesin motor family, which drives long-distance cargos along the MT 'tracts' in the anterograde direction using the energy generated by ATP hydrolysis $(48,49)$. Protrudin interacts with KIF5 $(16,49)$, thus influencing ER morphology in an MT-dependent mode. Furthermore, protrudin extracts lipids from the ER by interacting with PDZ domain-containing protein 8 (PDZD8), thus proving that these proteins coordinate with each other to influence ER morphology $(50,51)$. M1 spastin is able to interact with protrudin, thereby regulating ER morphology, probably by modulating the MT dynamics necessary for protrudin-mediated kinesin motor activities $(47,52)$.

Overall, spastin contributes to ER tubule formation and inhibits the SOCE process. Although the clear attribution of the ER sheet-tubule balance to cellular functions remains largely elusive, it is now known that $\mathrm{Ca}^{2+}$ signaling is affected. Furthermore, casein kinase 2 (CK2) is also activated upon spastin deficits (19). Whether other signaling pathways are activated or inhibited when the ER sheet-tubule balance is affected by spastin remains to be explored. Given that the ER is distributed throughout neurons, how spastin affects ER shaping, the calcium pathway and other signaling pathways in neural development and disease is an important issue in understanding the physiological and pathological process, which still requires further investigation. Answers to these questions will further elucidate the pathogenesis of HSP.
Spastin and LD formation. LDs are highly dynamic and complex organelles responsible for the assembly and storage of neutral lipids (53). They are specialized compartments of the tubular ER, from which they are generated in a stepwise process (54). Lipids from LDs are transported to the cell membranes and regulate intracellular signals $(55,56)$. Various studies postulated that spastin anchors to LDs through its hydrophobic domain (57-86). Spastin depletion or overexpression of the dominant-negative variant impairs the formation of LDs in the nerves and skeletal muscles of fruit flies, while overexpression of spastin promotes the formation of LDs $(11,57)$. These results suggest that $\mathrm{M} 1$ spastin is associated with the formation of LDs. Although there is no direct evidence indicating how spastin affects the formation of LDs, it may be hypothesized that the newly formed LDs may be ER shaping products, as LDs are specialized compartments of the tubular ER. However, how LDs function remains largely elusive. Whether LDs transported to the cell membrane or intracellular signals are changed still requires further research. In addition, it has not been demonstrated that dysfunction of LDs is involved in HSP pathogenesis. Whether LD abnormalities are a common defect or rather a readout of different dysfunctional pathways remains elusive. Therefore, LD function should be investigated in patients with HSP to further the understanding of HSP and offer novel therapeutic opportunities.

Interaction between the MIT domain and ESCRT-III. The MIT domain is adequate for binding two ESCRT-III proteins: increased sodium tolerance-1 (IST1) and charged multivesicular body protein 1B (CHMP1B) $(17,58,59)$. This interaction allows the recruitment of spastin to specific subcellular localizations, thus regulating spatial MT dynamics. The ESCRT-III machinery is vital for membrane scission in numerous membrane remodeling processes, including viral budding from the cell surface, budding of endosomes to form vesicles of late endosomes and cytokinetic abscission (14,60,61). In addition, the ESCRT-III proteins recruit spastin to regulate cytokinetic abscission, endosomal trafficking and fatty acid (FA) metabolism.

Cytokinetic abscission. The role of spastin during cytokinesis has been extensively elucidated and reviewed in various studies $(30,58,62)$. In the present review, the process is briefly discussed. During the late stages of the anaphase, the nuclear envelope, which is frequently intersected by spindle MTs, is progressively reassembled around the reforming daughter nuclei. In this process, spastin recruitment by IST1 helps to remove the remaining spindle MTs for nuclear envelope sealing. Failure of this process leads to the accumulation of DNA damage.

Similarly, the daughter cells remain connected by an intercellular bridge with the midbody ring overlapping with MTs at its center during the late stages of abscission. CHMP1B thus recruits spastin to remove the MTs in the intercellular bridge. Depletion of spastin results in abscission failure, causing the daughter cells to remain connected by elongated MT-filled bridges.

Endosomal trafficking. Endosomes are sorting organelles that mediate the internalization of extracellular transmembrane molecules and ligands. Endosomes are categorized into early, recycling and late endosomes based on their internalization 
stage. Internalized molecules may be recycled into the cell membrane using recycling endosomes or degraded using late endosomes $(63,64)$. Endosomal trafficking regulates plasma membrane receptor concentrations, which are critical extracellular responses. The ESCRT machinery recruits spastin into endosomes to regulate endosome fission and trafficking pathways $(65,66)$.

Existing evidence suggests that spastin regulates endosome trafficking in two ways. i) Spastin may promote the tubulation and fission of endosomes, thereby inhibiting their pathway to the lysosome $(17,18,67)$. This process is accomplished by the actin-dependent and MT-dependent pulling force and membrane scission by dynamin $(68,69)$. In this process, the ESCRT-III protein increased sodium tolerance 1 recruits spastin into the endosome through the interaction of its MIT domain. Previously, severing activities mediated by spastin were thought to generate more MTs (38), thus ensuring that MTs were loaded with more dynein to enhance the driving force, thereby resulting in an enhancement of endosome tabulation and fission. However, this point of view lacks experimental evidence. Of note, a recent study provided direct evidence that the ER and endosome membrane contact sites (MCSs) are vital for endosome tubulation and fission (18). Overall, a better explanation for the tubulation and fission of endosomes may be the result of spastin-mediated ER reorganization. The changed ER network reconstructs the MCSs and contributes to promoting endosome tabulation and fission, while how spastin affects the MCSs between the ER and endosomes remains to be elucidated. ii) Spastin is able to regulate endosome trafficking by antagonizing protrudin, thereby regulating endosome trafficking in a generalized manner (16). Protrudin is an ER-resident protein that interacts with Rab7 and phosphatidylinositol 3-phosphate. These interactions facilitate the transfer of KIF5 to the late endosomal motor adaptor FYVE and coiled-coil domain containing 1 , thus promoting endosomal transport to the plasma membrane $(70,71)$. Although the interaction of spastin with KIF5 remains to be proven, studies have indicated that it is able to interact with protrudin (52). Regardless of whether it is direct or indirect, this interaction may recruit spastin to KIF5, resulting in MT 'railway breakage', which blocks the late endosome toward the cell periphery.

To date, the process of endosomal behaviors mediated by spastin has remained largely elusive. As described above, spastin promotes endosome tabulation and fission to inhibit the lysosome pathway. Spastin regulates endosome trafficking in a generalized manner. Of note, the destination of the endosome cargo remains to be determined. Taking BMP receptors as an example, it is unlikely that the BMP receptor retransports to the cell periphery, as its level on the membrane increases upon deficits of spastin. Furthermore, spastin inhibits the lysosomal pathway, and it remains to be determined how cargo is metabolized in the cell. In addition, the specific cargos transported through spastin-mediated endosomal trafficking remain to be identified. Answering these questions may lead to the elucidation of the specific mechanisms of endosomal trafficking mediated by spastin and pave the road for the development of therapies for HSP. With the rapid progression of biological analysis techniques, it may be promising to identify endosome components and trace these cargos, thus elucidating the endosomal trafficking mediated by spastin and revealing the molecular and cellular pathogenic mechanisms leading to HSP.

LD-peroxisome tethering. Membrane contact sites between LDs and peroxisomes have been observed for decades. However, the molecular underpinning that connects these organelles remains largely elusive $(72,73)$. Peroxisomes harvest FAs from LDs for energy production and maintain cellular homeostasis by recycling lipids and protecting cells from oxidative stress and damage $(74,75)$. It has been postulated that spastin tethers to peroxisomes through the interaction between the MIT domain and the peroxisome resident protein ATP-binding cassette sub-family D member 1 and mediates FA flux from LDs to peroxisomes (13). Furthermore, overexpression of M1 spastin reduces peroxidized lipid accumulation in cells exposed to oxidative stress. On the other hand, the dominant-negative variant mutant spastin (K388R) is not able to promote LD peroxisome tethering and fails to lower peroxidized lipid levels in LDs $(13,76)$. In addition, HSP patient-derived cells with mutations in spastin also indicated impaired peroxisome movement and distribution. These observations have established an intuitionistic model to describe the mechanism of cellular organelle crosstalk between LDs and peroxisomes and the process of FA trafficking and metabolism. However, there is still a lack of evidence that FA impairment is involved in patients with HSP, which requires further validation. Overall, this mechanism strongly suggests that LD peroxisome tethering has an important role in the neuropathology of HSP and may provide an opportunity of targeting FA metabolism for HSP therapy.

\section{Regulation of spastin's stability and activity}

Spastin's stability and activity are spatiotemporally regulated at different transcriptional levels and with posttranslational modifications and coordination of other MAPs. Understanding the precise mechanisms involved in regulating spastin protein levels and activity may provide novel therapeutic targets for HSP based on haploinsufficiency $(77,78)$. To date, no relevant spastin regulators have been identified in patients with HSP. However, numerous factors have been reported to regulate its stability and activity in in vitro cell models. For instance, numerous transcription factors (NRF1 and SOX11) and microRNAs (miRs), including miR-30, -33a, -96 and -182, are able to regulate the expression levels of spastin (79-81). At the posttranscriptional level, spastin may be phosphorylated at S268 and recruited to the midbody (62); SUMOylation of spastin at K427 may alter the severing ability of spastin (26); ubiquitination of spastin at K554 mediates degradation (82). In addition, the consolidation of protein on MT (Tau, collapsin response mediator protein 5) also regulates the severing activity of spastin $(83,84)$. The Dpy-30 histone methyltransferase complex regulatory subunit gene, which modifies in hereditary spastic paraplegia, has an epistatic interaction with spastin (85). Understanding spastin's regulatory mechanisms may help identify novel therapeutic strategies against HSP. Table I highlights the specific regulators and their impact on spastin. Further putative regulators of spastin should be identified through proteomic and genomic approaches to understand 
Table I. Spastin's stability and activity regulators.

Regulators

Function

(Refs.)

Transcription factors

NRF1, SOX11 Positive regulation of spastin expression

Posttranscriptional regulators

miR96, miR-182, miR-30, miR-33a Negative regulation of spastin expression

Posttranscriptional modifications

Phosphorylation at S268

SUMOylation at K427

Ubiquitination at K554

Midbody localization to regulate abscission

Regulation of severing activity

Protein degradation

MAPs

Tau

Consolidation of this protein on MTs regulates the severing activity of spastin

CRMP5

Consolidation of this protein on MTs regulates the severing activity of spastin

Epistatic interaction

DYP30

Epistatic interaction with spastin during disease onset

NRF1, nuclear respiratory factor 1; MT, microtubule; MAP, MT-associated protein SOX11, SRY-box transcription factor 11; CRMP5, collapsin response-mediator protein-5; DYP30, Dpy-30 histone methyltransferase complex regulatory subunit; miR, microRNA.

the precise regulatory mechanisms of spastin in cellular activities.

\section{Role of spastin in neural development}

To date, no breakthrough has been made in the treatment of HSP-SPG4. However, a deeper understanding of the role of spastin in neural development contributes to more comprehensive insight into its etiology, thereby providing novel ideas for effective HSP-SPG4 treatment. Developing neurons require dynamic MTs to remodel their shapes and mediate kinesin and dynein-based cargo transport, thus promoting and maintaining axonal and dendritic processes $(86,87)$. The MT dynamics mediated by spastin in different subcellular locations highlight its vital role in neural development. The role of spastin in neural development has been gradually reported in recent years. In the chapter below, recent advances in spastin-mediated neurogenesis, axon elongation and branching, synapse formation and maturation, axon transportation and axon maintenance are discussed and existing problems that should be explored are highlighted. The roles of spastin during neural development are illustrated in Fig. 3.

Neurogenesis. Neurogenesis is active during the embryonic period, as it is responsible for producing new neurons. In this period, neural stem and progenitor cells go through symmetric proliferative division until a sufficient population of neural stem cells (NSCs) is produced and subsequently develops into neurons $(88,89)$. It has been reported that both M1 and M87 spastin is enriched in proliferative NSCs and embryonic brain tissues. Spastin depletion using short hairpin RNA led to a decrease in NSC proliferation and neuronal lineage differentiation. Of note, this phenomenon was partly reversed using the MT-destabilizing drug nocodazole, indicating that NSC proliferation requires the dynamic MTs provided by spastin (90). This observation suggests that spastin participates in neurogenesis by promoting MT dynamics. As the Sonic hedgehog (Shh) signaling pathway is responsible for regulating and coordinating cellular growth and development in the embryo, this study also revealed that the Shh signaling pathway was inhibited by spastin depletion (83). However, the inhibition mechanism of the Shh signaling pathway by spastin depletion should be further evaluated.

Axonal elongation and branching. Growth cones at the end of an axon mediate its extension and turning after axon differentiation. They sense the extracellular environment and regulate axonal development and turning. They also have a vital role in intercellular signaling transmission $(91,92)$. In general, axon development undergoes three stages: Protrusion, engorgement and consolidation. In the growth cone, the MTs are highly dynamic in contrast to the axonal draft. Highly dynamic MTs contribute to the high sensitivity of the cones to guidance cues. Furthermore, filopodia and lamellipodia consolidation also requires the mechanical force provided by dynamic MTs $(93,94)$.

MTs form highly stable and polarized bundles in axons, providing structural support and 'railways' to guide MT-dependent transport. During axon branching, the cytoskeleton must be remodeled, which requires actin filament accumulation to form axonal filopodia. Soon after that, the MTs then penetrate the actin-rich filopodia, allowing the maturation and continuous extension of the branches $(95,96)$. This phenomenon proves that MT dynamics are a vital factor during axon branching and formation.

As described above, M1 spastin is distributed in the cytosol and mainly at the membrane sites, such as the ER and LD. However, M87 spastin is distributed in the whole cell and was reported to be enriched in the growth cone (97), and part of the axonal branch points are likely to generate new branches $(24,98)$. There is abundant evidence suggesting that spastin contributes to axonal branching and elongation. 
A
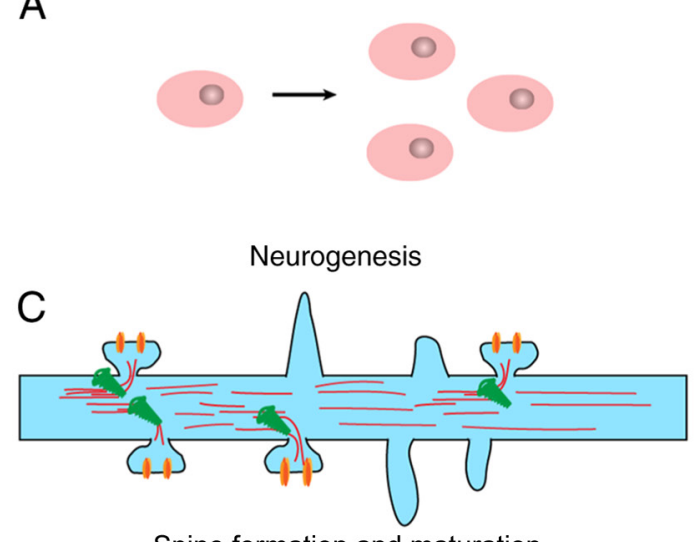

Spine formation and maturation

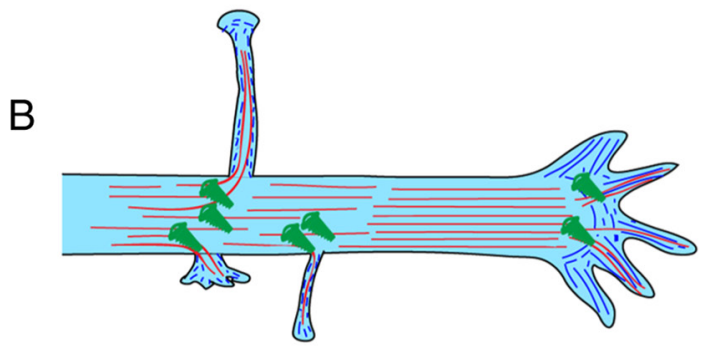

Axon branching and elongation

D

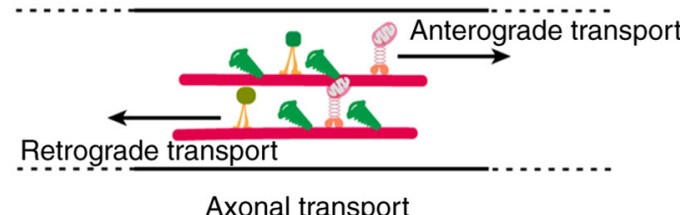

Axonal transport

\begin{tabular}{|lllllllllll}
\hline 0 & & & 0 & & & & & & & \\
NSC & MT & Actin & AMPAR & Dynein & Kinesin & Spastin & VAMP7 & BMP & Mitochondria \\
\hline
\end{tabular}

Figure 3. Roles of spastin during neural development. (A) Spastin promotes the proliferation of neural stem cells. (B) Spastin promotes axonal elongation and branch formation by generating more dynamic MTs that invade the growth cone of the filopodia and lamellipodia. (C) Spastin contributes to the formation and maturation of the dendritic spine by regulating AMPAR trafficking. (D) Spastin regulates axonal transport by altering the 'railway' of the motor protein. MT, microtubule; NSC, neural stem cell; AMPAR, $\alpha$-amino-3-hydroxy-5-methyl-4-isoxazolepropionic acid receptor; VAMP7, vesicle-associated membrane protein 7; BMP, bone morphogenetic protein-2.

Furthermore, the new MT severing theory suggests that spastin and other severing enzymes may act as amplifiers to generate more MT seeds during regrowth $(29,38)$. Cumulative evidence has indicated that neurons are sensitive to severing activities. Appropriate severing by spastin and katanin, which are highly expressed in neurons during development, promotes axonal elongation (77,99-101). Of note, overexpression of spastin significantly promotes the formation of axonal branches, whereas katanin does not. The accumulation of spastin in the branch points strongly suggests that branch formation requires the accumulation of actin filaments and MTs (100). Thus, it may be inferred that spastin potentially affects the actin cytoskeleton by interacting with related actin-associated proteins through MIT and MTBD domains. However, the actual mechanism involved in spastin-mediated remodeling of the actin cytoskeleton should be further evaluated.

Dendritic spine formation and maturation. The formation and maturation of dendritic spines contribute to the connections between neighboring neurons, which is vital for responding to novel stimuli throughout neural development and adulthood $(102,103)$. In the last decade, research on spastin has primarily focused on axonal behavioral mechanisms. It is worth noting that M87 spastin is distributed in the entire cell compartment, including dendrites and dendritic spines $(26,100)$. How M87 spastin affects dendrite behaviors remains largely elusive.

A decade ago, spastin was reported to regulate MT dynamics during the elimination of neuromuscular junctions in fly knockout models (27). Recent studies further indicated that spastin is closely related to learning and memory. Furthermore, it participates in the formation and maturation of dendritic spines and regulates the transport of the $\alpha$-amino-3-hydroxy-5methyl-4-isoxazolepropionic acid receptor (AMPAR) subunit GluA1 (26) and GluA2 (25). Lopes et al (25) established a spastin-knockout mouse model and determined that the formation and maturation of dendritic spines were inhibited upon spastin depletion and that KIF5-mediated GluA2 transport was also blocked upon spastin depletion. AMPAR trafficking may induce long-term potential or long-term depression, thereby regulating the maturity of dendritic spines $(104,105)$. As such, spastin potentially regulates dendritic spine morphology by altering MT-dependent transport. However, another study suggested that overexpression of spastin leads to an increase in the proportion of immature dendritic spines and leads to a decrease in GluA1 membrane expression (26). That study also indicated that GluA1 trafficking is closely related to spastin-mediated endosomal trafficking. The different effects of spastin on GluA1 and GluA2 trafficking may be attributed to the different trafficking pathways. GluA1 membrane trafficking is primarily dependent on the endocytosis or exocytosis of endosomes, while GluA2 binds directly to glutamate receptor interacting protein (GRIP) and is then subjected to KIF5-mediated transport (106). Of note, GRIP is able to bind the GluA2 subunit but not to GluA1 or GluA4 (107). Furthermore, neural activities are sensitive to the dosage of spastin levels, and it still requires to be elucidated how spastin works during synapse formation under physiological conditions. Collectively, these studies postulate that spastin-mediated cargo delivery is closely related to the formation and maturation of dendritic spines by regulating AMPAR trafficking. Furthermore, studies have also confirmed that KIF5 is also able to transport GABA receptors toward the dendritic spine (108). However, whether spastin is able to regulate this process requires further exploration. 
Although the actin cytoskeleton is the primary regulator of spine morphology, MTs may also penetrate the spine transiently. Invasive MTs are able to transport relevant cargo proteins, such as p140Cap, to promote the polymerization of actin filaments (109). It may be assumed that spastin contributes to actin polymerization by interacting with actin-binding proteins, as it acts as an amplifier to generate more MT seeds during regrowth. However, this assumption should be verified.

Axonal transport and maintenance. Axon transport is essential for regulating axon composition, neural development, maintenance and survival $(110,111)$. The process is mediated by motor molecules (kinesin or dynein) to deliver different cargos, such as organelles, signal molecules and RNAs, along the MTs in an ATP-dependent manner. For instance, the kinesin motor mediates the anterograde transport responsible for delivering RNAs, proteins and organelles toward the growth cones and synapses. Similarly, dynein-mediated retrograde transport delivers nutrient factors and proteins that require degradation by the cell body, thus responding to the extracellular environment (112-114). This process is highly dependent on the MTs. As such, spastin is potentially involved, as it alters the MT dynamics and arrays.

Numerous studies have reported spastin involvement in MT-mediated cargo transportation. Of note, spastin mutations identified in patients with HSP lead to axonal swellings and axonal transport abnormalities $(19,20,115)$. To date, spastin has also been reported to regulate the transportation of mitochondria $(20,116)$, peroxisomes $(117,118)$, amyloid precursor protein (20), vesicle-associated membrane protein 7 (VAMP7) (119) and BMPII receptors (120). In particular, M1 spastin is crucial during fast axonal transport using isolated squid axoplasm but not M87 $(6,19)$. Consistent with these observations, M1 spastin has a pivotal role in VAMP7 transport in cortical neurons but not M87 (121). However, Connell et al (16) postulated that both M1 and M87 antagonize KIF5-mediated BMPII transport to the cell membrane. These studies confirm that both M1 and M87 spastin are involved in axonal transport despite the differences in each cargo transportation mechanism and their transport direction.

M1 and M87 are different in the process of axon transportation. M1 has an additional hairpin loop domain that determines its location in the ER and LDs (32). It was also reported that M1 spastin is more efficient in promoting endosomal tubule fission. (17). Thereafter, M1 spastin regulates cargo transportation in ER-dependent pathways, while M87 primarily directs cargo transport by altering MT dynamics. Furthermore, M1 spastin is able to potentially recruit M87 from the cytosolic pool, as spastin requires hexamerization to sever MTs (28). Therefore, it may be hypothesized that M1 is more efficient than M87 in axonal transport via ER-dependent pathways.

As described previously, M1 spastin contributes to tubular ER formation by regulating ER-surrounded MT dynamics. The ER is distributed throughout the whole cell and is conducive to the physical continuity of axons (122). The ER and other organelle membrane contact sites allow communication with each other, thereby regulating intracellular membrane trafficking $(123,124)$. For instance, Allison et al (18) revealed that spastin promotes endosomal fission and regulates its trafficking using ER-endosome MCSs. Furthermore, ER-resident proteins, such as protrudin and PDZD8, potentially contribute to endosome trafficking (50) after ER morphology is altered by M1 spastin. ER morphology alteration is closely associated with calcium flux $(125,126)$. The process is required to maintain axonal transport (126). Of note, spastin also potentially influences the calcium signaling pathway in axons, thus indirectly contributing to normal axonal transport. As such, the M1 spastin-mediated mechanism involved in the regulation of axonal transport may be ER-dependent, which requires further experimental confirmation.

\section{Role of spastin in HSP}

HSP is characterized by progressive weakness and paraplegia of the lower limbs. Its major pathological feature is corticospinal tract degeneration, which leads to axonal swellings, resulting in neurological disorders. A breakthrough in understanding this disease is that spastin was discovered to be an MT severing enzyme that acts as an MT amplifier to generate more MTs (90), thus providing a reasonable etiological basis for understanding spastin-induced HSP. To date, the major cause of HSP-SPG4 is postulated to be insufficient MT cutting caused by haploinsufficiency of spastin (127). However, haploinsufficiency alone cannot explain numerous phenomena observed in HSP-SPG4 models $(128,129)$. Furthermore, the gain-of-function scenario is also postulated to be one of the onsets of HSP $(130,131)$. Differences between spastin isoforms also add complexity to the understanding of the disease. The recent series of innovative studies on spastin provides a theoretical basis for understanding its pathogenic mechanisms. The present review primarily focuses on discussing the pathogenic mechanism of spastin combined with existing observations of spastin in cellular activities to elucidate how spastin mutations lead to HSP occurrence.

The haploinsufficiency scenario posits that the pathogenesis of HSP-SPG4 is a consequence of decreased functional spastin protein due to the presence of the mutant protein, which cannot function normally (132). However, gain-of-function is another different mechanism. It is proposed that mutant spastin aggregates and elicits toxicity. One of the most obvious features is that a small number of spastin protein mutations may lead to negative effects. This phenomenon is supported by evidence indicating that spastin mutants activate CK2.CK2 phosphorylates both kinesin-1 and dynein, which mediate cargo release from the motor (19). A recent review postulated that gain-of-function may be the primary cause of HSP-SPG4 pathogenesis, while haploinsufficiency makes axons more vulnerable to a second insult (31).

With the increase in the current knowledge of spastin, the molecular and cellular mechanisms have been revealed by intense studies. These mechanisms contribute to a comprehensive understanding of this disease. It may be suggested that haploinsufficiency and gain-of-function are not controversial but complementary to each other, causing HSP. Furthermore, numerous gain-of-function observations may be explained from the current literature and require further exploration. A more reasonable explanation combined with recent discoveries of the molecular and cellular mechanisms of spastin may thus be proposed.

First, the gain-of-function scenario is supported by evidence that M1 mutants cause hyperactivation of CK2, 
which is an effect not shared with the M87 mutant (19). However, there is no direct evidence of how M1 spastin activates CK2. As described above, M1 spastin mutants lead to ER shaping abnormalities. Furthermore, CK2 activation in numerous cell types, including neurons, is closely associated with ER stress (133-135). Therefore, it is conjectured that the insufficient ER surrounding MT severing mediated by the mutant spastin potentially leads to indirect CK2 activation. The gain-of-function phenomenon is also supported by MT destabilization caused by the mutant spastin. Second, the gain-of-function scenario is also supported by the fact that mutant spastin may result in MT destabilization. Regarding this phenomenon, MT destabilization, referring to a decreased proportion of acetylated and detyrosinated tubulin, was detected by western blot analysis (130). Combined with the severing model described above, spastin deficits may lead to a loss of MTs, as spastin acts as an MT amplifier under physiological conditions. Finally, the gain-of-function scenario is further supported by knockout and knockdown models. Spastin knockout and knockdown mice have only a mild phenotype of axonal swellings. For instance, SPAST ${ }^{\mathrm{C} 448 \mathrm{Y}}$ mice exhibit locomotor phenotypes far more reminiscent of HSP than any other mouse despite corticospinal degeneration (130). From our perspective, this is not controversial, as the knockout and knockdown mice would have compensatory mechanisms and other related proteins, such as REEP1 and alastin, to rescue the phenotype caused by insufficient spastin dosage. The ER sites in SPAST ${ }^{\mathrm{C} 448 \mathrm{Y}}$ mice may be occupied by mutant spastin, thus inhibiting other rescue effects through the dominant-negative mechanism. However, whether the expression of other proteins is altered in spastin knockout or knockdown mice should be further examined.

Another question that should be addressed is whether M1 or M87 mutations cause the occurrence of HSP. Axonal transport impairment is documented as the main HSP feature associated with spastin mutations. Regarding whether M1 or M87 induce the impairment of axonal transport, Solowska et al (6) demonstrated that axonal transport is affected by M1 spastin but not M87 by using squid axoplasm with different HSP-related mutant spastin. Furthermore, M1 spastin is highly expressed in the corticospinal tract, which is consistent with HSP pathology $(6,19)$. Cognizant of this, M1 mutation appears to be more relevant to HSP. In addition, M1 mutation was markedly more toxic than mutant M87 to the effects of motor neurons in transgenic flies and neurite outgrowth in primary cortical neurons (131). However, studies postulate that the vast majority of spastin disease mutations affect both protein isoforms. Connell et al (16) and Allison et al (18) provided a reasonable explanation for the relationship between M1 and M87 isoforms based on the coordination of spastin-mediated endosome trafficking. They hypothesized that M1 localizes and nucleates in the ER and subsequently binds with M87 by recruiting it from the cytosolic pool to form functional hexamers. This observation was further supported by the evidence that M1 is more efficient than M87 in endosome tubule fission. Collectively, both spastin isoforms are involved in HSP pathogenesis, with M1 mutations possibly being more efficient than M87 mutations.

Understanding the etiology of HSP is vital in designing appropriate therapeutic strategies for patients with HSP. At present, HSP-SPG4 is mostly thought to be mediated by haploinsufficiency and insufficient MT severing. Null spastin homologs have been used to identify drugs that are able to reverse the HSP phenotype (128). For instance, the application of phenazine, methylene blue and $\mathrm{N}$-acetyl-cysteine improves locomotor activity. These compounds have been approved by the Food and Drug Administration and hold great promise for translation to therapy (1). In addition, vinblastine and nocodazole are able to abrogate axonal swellings associated with spastin-induced impaired axonal transport in cortical neurons of knockout mice. However, application of these drugs would cause side effects (136). Furthermore, overexpression of M1 or M87 spastin isoforms restores neurite length, branching and the number of primary neurites and reduces swelling in neuronal cells (10). However, this strategy, combined with other approaches, should be empirically designed and tested in animal models prior to roll-out. As such, targeting drugs for HSP-SPG4 remains an area of focus for future extensive studies.

\section{Concluding remarks}

HSP is a disease caused by different gene mutations with diverse phenotypes. However, the onset of HSP is usually caused by a single gene mutation. Therefore, it is promising to develop precise drugs for targeted treatment of single genes. Spastin is the most common mutated gene of HSP, accounting for $60 \%$ of autosomal dominant inheritance and $15 \%$ of sporadic cases. Taking HSP-SPG4 as a starting point, research on spastin in the past two decades has revealed numerous underlying molecular mechanisms in cell function, aiding in understanding the potential mechanism of axon degeneration. However, the cellular functions of spastin remain to be fully clarified and gene therapy targeting spastin for HSP is still full of challenges.

The key discovery to understand spastin's function is that it is an MT-severing enzyme that is able to cut long MTs into short fragments. Spastin is a protein with multiple modular domains. By interacting with other proteins or membrane structures, spastin is able to bring severing activity to various cellular subregions and participate in different cellular activities, such as endosomal transport, ER shaping, MT-based transport and lipid metabolism. However, their molecular mechanisms have yet to be fully elucidated. In addition, certain innovative cellular mechanisms mediated by spastin, such as LD formation and peroxisome-mediated FA metabolism in patients with HSP, remain to be explored. High-resolution image visualization technology is required to observe the ultramicrocellular structure of axon degeneration in patients or models of HSP.

Although devising drugs targeting spastin appears to be more promising, there are still numerous obstacles that hinder the development of precision therapeutic drugs. First, the etiology of HSP-SPG4 remains elusive. Specifically, it remains to be clarified whether haploinsufficiency or gain-of-function cause the degeneration of axons. If the gain-of-function mechanism truly exists, the toxic mutant spastin is imperative to be removed or the toxic effects caused by mutant spastin should be inhibited. For instance, using CK2 inhibitor to activate the axonal transport could reduce the axonal swellings in HSP patients. Furthermore, it remains to be determined whether M1 and M87 mutations mediate the occurrence of HSP 
independently or coordinatively and how M1 and M87 coordinate to function. Both isoforms are able to restore neurite length and branching and reduce axon swellings in induced pluripotent stem cells from a patient with a spastin nonsense mutation, and it remains to be determined which isoform or whether both of them are vital for recovery. It should also be clarified what isoform should be used as a therapeutic target. In addition, the use of MT depolymerization drugs such as vinblastine improves the phenotype of HSP-SPG4, but MT depolymerization drugs are not targeted. It remains to be elucidated whether they change the MT dynamics in other cellular regions and cause other side effects. Finally, excessive spastin destroys the cytoskeleton and is toxic to neurons. It is thus required to determine how to administer the accurate dosage of spastin according to physiological conditions to reach the axon degeneration site.

Fast progress in understanding the molecular mechanisms of spastin holds promise to unveil the most basic cellular biological mechanism of HSP-SPG4 in the near future. Resolving these questions will help provide more precise and innovative treatments for patients with HSP.

\section{Acknowledgements}

Not applicable.

\section{Funding}

This work was supported by the National Natural Science Foundation of China (grant nos. 31300885, 32170977, 82102314 and 81771311), Project of Educational Commission of Guangdong Province of China (grant no. 2018KQNCX013), the Fundamental Research Funds for the Central Universities Project (grant no. 11618304) and the China Postdoctoral Science Foundation (grant no. 2019M653292).

\section{Availability of data and materials}

Data sharing is not applicable to this article, as no datasets were generated or analyzed during the current study.

\section{Authors' contributions}

QLL performed the literature search and wrote the manuscript. GWZ contributed to the molecular mechanisms. ZSJ and HSL provided supervision and revised the manuscript. All authors read and approved the final manuscript. Data authentication is not applicable.

\section{Ethics approval and consent to participate}

Not applicable.

\section{Patient consent for publication}

Not applicable.

\section{Competing interests}

The authors declare that they have no competing interests.

\section{References}

1. Shribman S, Reid E, Crosby AH, Houlden H and Warner TT: Hereditary spastic paraplegia: From diagnosis to emerging therapeutic approaches. Lancet Neurol 18: 1136-1146, 2019.

2. Schüle R, Wiethoff S, Martus P, Karle KN, Otto S, Klebe S, Klimpe S, Gallenmüller C, Kurzwelly D, Henkel D, et al: Hereditary spastic paraplegia: Clinicogenetic lessons from 608 patients. Ann Neurol 79: 646-658, 2016.

3. Solowska JM and Baas PW: Hereditary spastic paraplegia SPG4: What is known and not known about the disease. Brain 138: 2471-2484, 2015.

4. Fink JK: Hereditary spastic paraplegia: Clinico-pathologic features and emerging molecular mechanisms. Acta Neuropathol 126: 307-328, 2013.

5. Salinas S, Carazo-Salas RE, Proukakis C, Schiavo G and Warner TT: Spastin and microtubules: Functions in health and disease. J Neurosci Res 85: 2778-2782, 2007.

6. Solowska JM, Morfini G, Falnikar A, Himes BT, Brady ST, Huang D and Baas PW: Quantitative and functional analyses of spastin in the nervous system: Implications for hereditary spastic paraplegia. J Neurosci 28: 2147-2157, 2008.

7. Deluca GC, Ebers GC and Esiri MM: The extent of axonal loss in the long tracts in hereditary spastic paraplegia. Neuropathol Appl Neurobiol 30: 576-584, 2004.

8. Lumb JH, Connell JW, Allison R and Reid E: The AAA ATPase spastin links microtubule severing to membrane modelling. Biochim Biophys Acta 1823: 192-197, 2012.

9. Schickel J, Pamminger T, Ehrsam A, Münch S, Huang X, Klopstock T, Kurlemann G, Hemmerich P, Dubiel W, Deufel T and Beetz C: Isoform-specific increase of spastin stability by $\mathrm{N}$-terminal missense variants including intragenic modifiers of SPG4 hereditary spastic paraplegia. Eur J Neurol 14: 1322-1328, 2007.

10. Havlicek S, Kohl Z, Mishra HK, Prots I, Eberhardt E, Denguir N, Wend H, Plötz S, Boyer L, Marchetto MC, et al: Gene dosage-dependent rescue of HSP neurite defects in SPG4 patients' neurons. Hum Mol Genet 23: 2527-2541, 2014.

11. Arribat Y, Grepper D, Lagarrigue S, Qi T, Cohen S and Amati F: Spastin mutations impair coordination between lipid droplet dispersion and reticulum. PLoS Genet 16: e1008665, 2020.

12. Park SH, Zhu PP, Parker RL and Blackstone C: Hereditary spastic paraplegia proteins REEP1, spastin, and atlastin-1 coordinate microtubule interactions with the tubular ER network. J Clin Invest 120: 1097-1110, 2010.

13. Chang CL, Weigel AV, Ioannou MS, Pasolli HA, Xu CS, Peale DR, Shtengel G, Freeman M, Hess HF, Blackstone C, et al: Spastin tethers lipid droplets to peroxisomes and directs fatty acid trafficking through ESCRT-III. J Cell Biol 218: 2583-2599, 2019.

14. Vietri M, Radulovic M and Stenmark H: The many functions of ESCRTs. Nat Rev Mol Cell Biol 21: 25-42, 2020.

15. Guo EZ and Xu Z: Distinct mechanisms of recognizing endosomal sorting complex required for transport III (ESCRT-III) protein IST1 by different microtubule interacting and trafficking (MIT) domains. J Biol Chem 290: 8396-8408, 2015.

16. Connell JW, Allison RJ, Rodger CE, Pearson G, Zlamalova E and Reid E: ESCRT-III-associated proteins and spastin inhibit protrudin-dependent polarised membrane traffic. Cell Mol Life Sci 77: 2641-2658, 2020.

17. Allison R, Lumb JH, Fassier C, Connell JW, Ten Martin D, Seaman MN, Hazan J and Reid E: An ESCRT-spastin interaction promotes fission of recycling tubules from the endosome. J Cell Biol 202: 527-543, 2013.

18. Allison R, Edgar JR, Pearson G, Rizo T, Newton T, Günther S, Berner F, Hague J, Connell JW, Winkler J, et al: Defects in ER-endosome contacts impact lysosome function in hereditary spastic paraplegia. J Cell Biol 216: 1337-1355, 2017.

19. Leo L, Weissmann C, Burns M, Kang M, Song Y, Qiang L, Brady ST, Baas PW and Morfini G: Mutant spastin proteins promote deficits in axonal transport through an isoform-specific mechanism involving casein kinase 2 activation. Hum Mol Genet 26: 2321-2334, 2017.

20. Kasher PR, De Vos KJ, Wharton SB, Manser C, Bennett EJ, Bingley M, Wood JD, Milner R, McDermott CJ, Miller CC, et al: Direct evidence for axonal transport defects in a novel mouse model of mutant spastin-induced hereditary spastic paraplegia (HSP) and human HSP patients. J Neurochem 110: 34-44, 2009. 
21. Jeong B, Kim TH, Kim DS, Shin WH, Lee JR, Kim NS and Lee DY: Spastin contributes to neural development through the regulation of microtubule dynamics in the primary cilia of neural stem cells. Neuroscience 411: 76-85, 2019.

22. Goyal U, Renvoisé B, Chang J and Blackstone C: Spastin-interacting protein NA14/SSNA1 functions in cytokinesis and axon development. PLoS One 9: e112428, 2014.

23. Ji Z, Zhang G, Chen L, Li J, Yang Y, Cha C, Zhang J, Lin H and Guo G: Spastin interacts with CRMP5 to promote neurite outgrowth by controlling the microtubule dynamics. Dev Neurobiol 78: 1191-1205, 2018.

24. Wood JD, Landers JA, Bingley M, McDermott CJ, Thomas-McArthur V, Gleadall LJ, Shaw PJ and Cunliffe VT: The microtubule-severing protein Spastin is essential for axon outgrowth in the zebrafish embryo. Hum Mol Genet 15: 2763-2771, 2006.

25. Lopes AT, Hausrat TJ, Heisler FF, Gromova KV, Lombino FL, Fischer T, Ruschkies L, Breiden P, Thies E, Hermans-Borgmeyer I, et al: Spastin depletion increases tubulin polyglutamylation and impairs kinesin-mediated neuronal transport, leading to working and associative memory deficits. PLoS Biol 18: e3000820, 2020.

26. Ji ZS, Liu QL, Zhang JF, Yang YH, Li J, Zhang GW, Tan MH, Lin HS and Guo GQ: SUMOylation of spastin promotes the internalization of GluA1 and regulates dendritic spine morphology by targeting microtubule dynamics. Neurobiol Dis 146: 105133, 2020.

27. Sherwood NT, Sun Q, Xue M, Zhang B and Zinn K: Drosophila spastin regulates synaptic microtubule networks and is required for normal motor function. PLoS Biol 2: e429, 2004.

28. Roll-Mecak A and Vale RD: Structural basis of microtubule severing by the hereditary spastic paraplegia protein spastin. Nature 451: 363-367, 2008.

29. Kuo YW, Trottier O, Mahamdeh M and Howard J: Spastin is a dual-function enzyme that severs microtubules and promotes their regrowth to increase the number and mass of microtubules. Proc Natl Acad Sci USA 116: 5533-5541, 2019.

30. Connell JW, Lindon C, Luzio JP and Reid E: Spastin couples microtubule severing to membrane traffic in completion of cytokinesis and secretion. Traffic 10: 42-56, 2009.

31. Qiang L, Piermarini E and Baas PW: New hypothesis for the etiology of SPAST-based hereditary spastic paraplegia. Cytoskeleton (Hoboken) 76: 289-297, 2019.

32. Sakoe K, Shioda N and Matsuura T: A newly identified NES sequence present in spastin regulates its subcellular localization and microtubule severing activity. Biochim Biophys Acta Mol Cell Res 1868: 118862, 2021

33. Beetz C, Brodhun M, Moutzouris K, Kiehntopf M, Berndt A, Lehnert D, Deufel T, Bastmeyer M and Schickel J: Identification of nuclear localisation sequences in spastin (SPG4) using a novel Tetra-GFP reporter system. Biochem Biophys Res Commun 318: 1079-1084, 2004.

34. Monteonofrio L, Valente D, Rinaldo C and Soddu S: Extrachromosomal Histone H2B contributes to the formation of the abscission site for cell division. Cells 8: 1391, 2019.

35. Sandate CR, Szyk A, Zehr EA, Lander GC and Roll-Mecak A: An allosteric network in spastin couples multiple activities required for microtubule severing. Nat Struct Mol Biol 26: 671-678, 2019.

36. Han H, Schubert HL, McCullough J, Monroe N, Purdy MD Yeager M, Sundquist WI and Hill CP: Structure of spastin bound to a glutamate-rich peptide implies a hand-over-hand mechanism of substrate translocation. J Biol Chem 295: 435-443, 2020.

37. White SR, Evans KJ, Lary J, Cole JL and Lauring B: Recognition of C-terminal amino acids in tubulin by pore loops in Spastin is important for microtubule severing. J Cell Biol 176: 995-1005, 2007.

38. Vemu A, Szczesna E, Zehr EA, Spector JO, Grigorieff N Deaconescu AM and Roll-Mecak A: Severing enzymes amplify microtubule arrays through lattice GTP-tubulin incorporation. Science 361: eaau1504, 2018

39. Kuo YW, Trottier O and Howard J: Predicted effects of severing enzymes on the length distribution and total mass of microtubules. Biophys J 117: 2066-2078, 2019.

40. Saltini M and Mulder BM: Critical threshold for microtubule amplification through templated severing. Phys Rev E 101: 052405,2020

41. Rao K, Stone MC, Weiner AT, Gheres KW, Zhou C, Deitcher DL, Levitan ES and Rolls MM: Spastin, atlastin, and ER relocalization are involved in axon but not dendrite regeneration. Mol Biol Cell 27: 3245-3256, 2016
42. Vajente N, Norante R, Redolfi N, Daga A, Pizzo P and Pendin D: Microtubules stabilization by mutant spastin affects ER morphology and $\mathrm{Ca}^{2+}$ handling. Front Physiol 10: 1544, 2019.

43. Pendin D, McNew JA and Daga A: Balancing ER dynamics: Shaping, bending, severing, and mending membranes. Curr Opin Cell Biol 23: 435-442, 2011

44. Farías GG, Fréal A, Tortosa E, Stucchi R, Pan X, Portegies S, Will L, Altelaar M and Hoogenraad CC: Feedback-driven mechanisms between microtubules and the endoplasmic reticulum instruct neuronal polarity. Neuron 102: 184-201.e8, 2019.

45. Liu X, Guo X, Niu L, Li X, Sun F, Hu J, Wang X and Shen K: Atlastin-1 regulates morphology and function of endoplasmic reticulum in dendrites. Nat Commun 10: 568, 2019.

46. Hashimoto Y, Shirane M, Matsuzaki F, Saita S, Ohnishi T and Nakayama KI: Protrudin regulates endoplasmic reticulum morphology and function associated with the pathogenesis of hereditary spastic paraplegia. J Biol Chem 289: 12946-12961, 2014.

47. Chang J, Lee S and Blackstone C: Protrudin binds atlastins and endoplasmic reticulum-shaping proteins and regulates network formation. Proc Natl Acad Sci USA 110: 14954-14959, 2013.

48. Iworima DG, Pasqualotto BA and Rintoul GL: Kif5 regulates mitochondrial movement, morphology, function and neuronal survival. Mol Cell Neurosci 72: 22-33, 2016.

49. Matsuzaki F, Shirane M, Matsumoto M and Nakayama KI Protrudin serves as an adaptor molecule that connects KIF5 and its cargoes in vesicular transport during process formation. Mol Biol Cell 22: 4602-4620, 2011

50. Shirane M, Wada M, Morita K, Hayashi N, Kunimatsu R, Matsumoto Y, Matsuzaki F, Nakatsumi H, Ohta K, Tamura Y and Nakayama KI: Protrudin and PDZD8 contribute to neuronal integrity by promoting lipid extraction required for endosome maturation. Nat Commun 11: 4576, 2020.

51. Shirane M: Lipid transfer-dependent endosome maturation mediated by protrudin and PDZD8 in neurons. Front Cell Dev Biol 8: 615600, 2020.

52. Zhang C, Li D, Ma Y, Yan J, Yang B, Li P, Yu A, Lu C and Ma X: Role of spastin and protrudin in neurite outgrowth. J Cell Biochem 113: 2296-2307, 2012.

53. Olzmann JA and Carvalho P: Dynamics and functions of lipid droplets. Nat Rev Mol Cell Biol 20: 137-155, 2019.

54. Walther TC, Chung J and Farese RV Jr: Lipid droplet biogenesis. Annu Rev Cell Dev Biol 33: 491-510, 2017.

55. Welte MA and Gould AP: Lipid droplet functions beyond energy storage. Biochim Biophys Acta Mol Cell Biol Lipids 1862: 1260-1272, 2017.

56. Velázquez AP, Tatsuta T, Ghillebert R, Drescher I and Graef M: Lipid droplet-mediated ER homeostasis regulates autophagy and cell survival during starvation. J Cell Biol 212: 621-631, 2016.

57. Papadopoulos C, Orso G, Mancuso G, Herholz M, Gumeni S, Tadepalle N, Jüngst C, Tzschichholz A, Schauss A, Höning S, et al: Spastin binds to lipid droplets and affects lipid metabolism. PLoS Genet 11: e1005149, 2015.

58. Vietri M, Schink KO, Campsteijn C, Wegner CS, Schultz SW Christ L, Thoresen SB, Brech A, Raiborg C and Stenmark H: Spastin and ESCRT-III coordinate mitotic spindle disassembly and nuclear envelope sealing. Nature 522: 231-235, 2015.

59. Reid E, Connell J, Edwards TL, Duley S, Brown SE and Sanderson CM: The hereditary spastic paraplegia protein spastin interacts with the ESCRT-III complex-associated endosomal protein CHMP1B. Hum Mol Genet 14: 19-38, 2005.

60. Christ L, Raiborg C, Wenzel EM, Campsteijn C and Stenmark H: Cellular functions and molecular mechanisms of the ESCRT membrane-scission machinery. Trends Biochem Sci 42: 42-56, 2017.

61. Henne WM, Buchkovich NJ and Emr SD: The ESCRT pathway. Dev Cell 21: 77-91, 2011.

62. Pisciottani A, Biancolillo L, Ferrara M, Valente D, Sardina F, Monteonofrio L, Camerini S, Crescenzi M, Soddu S and Rinaldo C: HIPK2 phosphorylates the microtubule-severing enzyme spastin at S268 for abscission. Cells 8: 684, 2019.

63. Scott CC, Vacca F and Gruenberg J: Endosome maturation, transport and functions. Semin Cell Dev Biol 31: 2-10, 2014.

64. Tu Y, Zhao L, Billadeau DD and Jia D: Endosome-to-TGN trafficking: Organelle-vesicle and organelle-organelle interactions. Front Cell Dev Biol 8: 163, 2020.

65. Wang J, Fedoseienko A, Chen B, Burstein E, Jia D and Billadeau DD: Endosomal receptor trafficking: Retromer and beyond. Traffic 19: 578-590, 2018. 
66. Vagnozzi AN and Praticò D: Endosomal sorting and trafficking, the retromer complex and neurodegeneration. Mol Psychiatry 24: 857-868, 2019.

67. Allison R, Edgar JR and Reid E: Spastin MIT Domain Disease-Associated mutations disrupt lysosomal function. Front Neurosci 13: 1179, 2019.

68. Skjeldal FM, Strunze S, Bergeland T, Walseng E, Gregers TF and Bakke O: The fusion of early endosomes induces molecular-motor-driven tubule formation and fission. J Cell Sci 125: 1910-1919, 2012.

69. Hoyer MJ, Chitwood PJ, Ebmeier CC, Striepen JF, Qi RZ, Old WM and Voeltz GK: A novel class of ER membrane proteins regulates ER-associated endosome fission. Cell 175: 254-265.e14, 2018.

70. Raiborg C, Wenzel EM, Pedersen NM, Olsvik H, Schink KO, Schultz SW, Vietri M, Nisi V, Bucci C, Brech A, et al: Repeated ER-endosome contacts promote endosome translocation and neurite outgrowth. Nature 520: 234-238, 2015.

71. Elbaz-Alon Y, Guo Y, Segev N, Harel M, Quinnell DE, Geiger T, Avinoam O, Li D and Nunnari J: PDZD8 interacts with Protrudin and Rab7 at ER-late endosome membrane contact sites associated with mitochondria. Nat Commun 11: 3645, 2020.

72. Joshi AS, Nebenfuehr B, Choudhary V, Satpute-Krishnan P, Levine TP, Golden A and Prinz WA: Lipid droplet and peroxisome biogenesis occur at the same ER subdomains. Nat Commun 9: 2940, 2018.

73. Joshi AS and Cohen S: Lipid droplet and peroxisome biogenesis: Do they go hand-in-hand? Front Cell Dev Biol 7: 92, 2019.

74. Walker CL, Pomatto LCD, Tripathi DN and Davies KJA: Redox regulation of homeostasis and proteostasis in peroxisomes. Physiol Rev 98: 89-115, 2018.

75. Islinger M, Voelkl A, Fahimi HD and Schrader M: The peroxisome: An update on mysteries 2.0. Histochem Cell Biol 150: 443-471, 2018

76. Henne WM: Spastin joins LDs and peroxisomes in the interorganelle contact ballet. J Cell Biol 218: 2439-2441, 2019.

77. Riano E, Martignoni M, Mancuso G, Cartelli D, Crippa F, Toldo I, Siciliano G, Di Bella D, Taroni F, Bassi MT, et al: Pleiotropic effects of spastin on neurite growth depending on expression levels. J Neurochem 108: 1277-1288, 2009.

78. Denton KR, Lei L, Grenier J, Rodionov V, Blackstone C and Li XJ: Loss of spastin function results in disease-specific axonal defects in human pluripotent stem cell-based models of hereditary spastic paraplegia. Stem Cells 32: 414-423, 2014.

79. Henson BJ, Zhu W, Hardaway K, Wetzel JL, Stefan M, Albers KM and Nicholls RD: Transcriptional and post-transcriptional regulation of SPAST, the gene most frequently mutated in hereditary spastic paraplegia. PLoS One 7: e36505, 2012.

80. Jiang T, Cai Z, Ji Z, Zou J, Liang Z, Zhang G, Liang Y, Lin H and Tan M: The lncRNA MALAT1/miR-30/Spastin axis regulates hippocampal neurite outgrowth. Front Cell Neurosci 14: 555747, 2020.

81. Nakazeki F, Tsuge I, Horie T, Imamura K, Tsukita K, Hotta A, Baba O, Kuwabara Y, Nishino T, Nakao T, et al: MiR-33a is a therapeutic target in SPG4-related hereditary spastic paraplegia human neurons. Clin Sci (Lond) 133: 583-595, 2019.

82. Sardina F, Pisciottani A, Ferrara M, Valente D, Casella M, Crescenzi M, Peschiaroli A, Casali C, Soddu S, Grierson AJ and Rinaldo C: Spastin recovery in hereditary spastic paraplegia by preventing neddylation-dependent degradation. Life Sci Alliance 3: e202000799, 2020

83. Tan R, Lam AJ, Tan T, Han J, Nowakowski DW, Vershinin M, Simó S, Ori-McKenney KM and McKenney RJ: Microtubules gate tau condensation to spatially regulate microtubule functions. Nat Cell Biol 21: 1078-1085, 2019.

84. Jin Z, Shou HF, Liu JW, Jiang SS, Shen Y, Cheng WY and Gao LL: Spastin interacts with CRMP5 to promote spindle organization in mouse oocytes by severing microtubules. Zygote: $1-12,2021$.

85. Newton T, Allison R, Edgar JR, Lumb JH, Rodger CE, Manna PT, Rizo T, Kohl Z, Nygren AOH, Arning L, et al: Mechanistic basis of an epistatic interaction reducing age at onset in hereditary spastic paraplegia. Brain 141: 1286-1299, 2018.

86. Kapitein LC and Hoogenraad CC: Building the neuronal microtubule cytoskeleton. Neuron 87: 492-506, 2015.

87. Kelliher MT, Saunders HA and Wildonger J: Microtubule control of functional architecture in neurons. Curr Opin Neurobiol 57: 39-45, 2019.

88. Bond AM, Ming GL and Song H: Adult mammalian neural stem cells and neurogenesis: Five decades later. Cell Stem Cell 17: 385-395, 2015.
89. Katsimpardi L and Lledo PM: Regulation of neurogenesis in the adult and aging brain. Curr Opin Neurobiol 53: 131-138, 2018.

90. McNally FJ and Roll-Mecak A: Microtubule-severing enzymes: From cellular functions to molecular mechanism. J Cell Biol 217: 4057-4069, 2018.

91. Kahn OI and Baas PW: Microtubules and growth cones: Motors drive the turn. Trends Neurosci 39: 433-440, 2016.

92. Dent EW and Gertler FB: Cytoskeletal dynamics and transport in growth cone motility and axon guidance. Neuron 40: 209-227, 2003.

93. Lowery LA and Van Vactor D: The trip of the tip: Understanding the growth cone machinery. Nat Rev Mol Cell Biol 10: 332-343, 2009.

94. Dent EW, Gupton SL and Gertler FB: The growth cone cytoskeleton in axon outgrowth and guidance. Cold Spring Harb Perspect Biol 3: a001800, 2011.

95. Rao AN and Baas PW: Polarity sorting of microtubules in the axon. Trends Neurosci 41: 77-88, 2018.

96. Tas RP, Chazeau A, Cloin BMC, Lambers MLA, Hoogenraad CC and Kapitein LC: Differentiation between oppositely oriented microtubules controls polarized neuronal transport. Neuron 96: 1264-1271.e5, 2017.

97. Claudiani P, Riano E, Errico A, Andolfi G and Rugarli EI: Spastin subcellular localization is regulated through usage of different translation start sites and active export from the nucleus. Exp Cell Res 309: 358-369, 2005.

98. Butler R, Wood JD, Landers JA and Cunliffe VT: Genetic and chemical modulation of spastin-dependent axon outgrowth in zebrafish embryos indicates a role for impaired microtubule dynamics in hereditary spastic paraplegia. Dis Model Mech 3: 743-751, 2010

99. Karabay A, Yu W, Solowska JM, Baird DH and Baas PW: Axonal growth is sensitive to the levels of katanin, a protein that severs microtubules. J Neurosci 24: 5778-5788, 2004.

100. Yu W, Qiang L, Solowska JM, Karabay A, Korulu S and Baas PW: The microtubule-severing proteins spastin and katanin participate differently in the formation of axonal branches. Mol Biol Cell 19: 1485-1498, 2008.

101. Conde C and Cáceres A: Microtubule assembly, organization and dynamics in axons and dendrites. Nat Rev Neurosci 10 319-332, 2009

102. Herms J and Dorostkar MM: Dendritic spine pathology in neurodegenerative diseases. Annu Rev Pathol 11: 221-250, 2016.

103. Stein IS and Zito K: Dendritic spine elimination: Molecular mechanisms and implications. Neuroscientist 25: 27-47, 2019.

104. Park M: AMPA receptor trafficking for postsynaptic potentiation. Front Cell Neurosci 12: 361, 2018.

105. Chater TE and Goda Y: The role of AMPA receptors in postsynaptic mechanisms of synaptic plasticity. Front Cell Neurosci 8: 401, 2014.

106. Hanley JG: AMPA receptor trafficking pathways and links to dendritic spine morphogenesis. Cell Adh Migr 2: 276-282, 2008.

107. Dong H, O'Brien RJ, Fung ET, Lanahan AA, Worley PF and Huganir RL: GRIP: A synaptic PDZ domain-containing protein that interacts with AMPA receptors. Nature 386: 279-284, 1997.

108. Nakajima K, Yin X, Takei Y, Seog DH, Homma N and Hirokawa N: Molecular motor KIF5A is essential for GABA(A) receptor transport, and KIF5A deletion causes epilepsy. Neuron 76: 945-961, 2012.

109. Jaworski J, Kapitein LC, Gouveia SM, Dortland BR, Wulf PS, Grigoriev I, Camera P, Spangler SA, Di Stefano P, Demmers J, et al: Dynamic microtubules regulate dendritic spine morphology and synaptic plasticity. Neuron 61: 85-100, 2009.

110. Okabe S and Hirokawa N: Axonal transport. Curr Opin Cell Biol 1: 91-97, 1989.

111. Millecamps S and Julien JP: Axonal transport deficits and neurodegenerative diseases. Nat Rev Neurosci 14: 161-176, 2013.

112. Gibbs KL, Greensmith L and Schiavo G: Regulation of axonal transport by protein kinases. Trends Biochem Sci 40: 597-610, 2015.

113. Guedes-Dias P and Holzbaur ELF: Axonal transport: Driving synaptic function. Science 366: eaaw9997, 2019.

114. Cyr JL and Brady ST: Molecular motors in axonal transport. Cellular and molecular biology of kinesin. Mol Neurobiol 6: $137-155,1992$.

115. Fuerst JC, Henkel AW, Stroebel A, Welzel O, Groemer TW, Kornhuber J and Bönsch D: Distinct intracellular vesicle transport mechanisms are selectively modified by spastin and spastin mutations. J Cell Physiol 226: 362-368, 2011 
116. McDermott CJ, Grierson AJ, Wood JD, Bingley M, Wharton SB Bushby KM and Shaw PJ: Hereditary spastic paraparesis: Disrupted intracellular transport associated with spastin mutation. Ann Neurol 54: 748-759, 2003.

117. Wali G, Sutharsan R, Fan Y, Stewart R, Tello Velasquez J, Sue CM, Crane DI and Mackay-Sim A: Mechanism of impaired microtubule-dependent peroxisome trafficking and oxidative stress in SPAST-mutated cells from patients with Hereditary Spastic Paraplegia. Sci Rep 6: 27004, 2016.

118. Wali G,Liyanage E, Blair NF, Sutharsan R, Park JS, Mackay-Sim A and Sue CM: Oxidative stress-induced axon fragmentation is a consequence of reduced axonal transport in hereditary spastic paraplegia SPAST patient neurons. Front Neurosci 14: 401, 2020.

119. Plaud C, Joshi V, Marinello M, Pastré D, Galli T, Curmi PA and Burgo A: Spastin regulates VAMP7-containing vesicles trafficking in cortical neurons. Biochim Biophys Acta Mol Basis Dis 1863: 1666-1677, 2017

120. Jardin N, Giudicelli F, Ten Martín D, Vitrac A, De Gois S, Allison R, Houart C, Reid E, Hazan J and Fassier C: BMP- and neuropilin 1-mediated motor axon navigation relies on spastin alternative translation. Development 145: dev162701, 2018.

121. Plaud C, Joshi V, Kajevu N, Poüs C, Curmi PA and Burgo A: Functional differences of short and long isoforms of spastin harboring missense mutation. Dis Model Mech 11: dmm033704, 2018.

122. Öztürk Z, O'Kane CJ and Pérez-Moreno JJ: Axonal endoplasmic reticulum dynamics and its roles in neurodegeneration. Fron Neurosci 14: 48, 2020.

123. Henne WM, Liou J and Emr SD: Molecular mechanisms of inter-organelle ER-PM contact sites. Curr Opin Cell Biol 35 123-130, 2015.

124. Phillips MJ and Voeltz GK: Structure and function of ER membrane contact sites with other organelles. Nat Rev Mol Cell Biol 17: 69-82, 2016.

125. Chung WY, Jha A, Ahuja M and Muallem S: $\mathrm{Ca}^{2+}$ influx at the ER/PM junctions. Cell Calcium 63: 29-32, 2017.

126. Friel D: Interplay between ER $\mathrm{Ca}^{2+}$ uptake and release fluxes in neurons and its impact on $\left[\mathrm{Ca}^{2+}\right]$ dynamics. Biol Res 37: 665-674, 2004.

127. Rehbach K, Kesavan J, Hauser S, Ritzenhofen S, Jungverdorben J, Schüle R, Schöls L, Peitz M and Brüstle O: Multiparametric rapic screening of neuronal process pathology for drug target identification in HSP patient-specific neurons. Sci Rep 9: 9615, 2019.
128. Julien C, Lissouba A, Madabattula S, Fardghassemi Y, Rosenfelt C, Androschuk A, Strautman J, Wong C, Bysice A, O'sullivan $\mathrm{J}$, et al: Conserved pharmacological rescue of hereditary spastic paraplegia-related phenotypes across model organisms. Hum Mol Genet 25: 1088-1099, 2016.

129. Connell JW, Allison R and Reid E: Quantitative gait analysis using a motorized treadmill system sensitively detects motor abnormalities in mice expressing ATPase defective spastin. PLoS One 11: e0152413, 2016.

130. Qiang L, Piermarini E, Muralidharan H, Yu W, Leo L, Hennessy LE, Fernandes S, Connors T, Yates PL, Swift M, et al: Hereditary spastic paraplegia: Gain-of-function mechanisms revealed by new transgenic mouse. Hum Mol Genet 28: 1136-1152, 2019.

131. Solowska JM, D'Rozario M, Jean DC, Davidson MW, Marenda DR and Baas PW: Pathogenic mutation of spastin has gain-of-function effects on microtubule dynamics. J Neurosci 34: 1856-1867, 2014.

132. Yip AG, Dürr A, Marchuk DA, Ashley-Koch A, Hentati A, Rubinsztein DC and Reid E: Meta-analysis of age at onset in spastin-associated hereditary spastic paraplegia provides no evidence for a correlation with mutational class. J Med Genet 40: e106, 2003.

133. Wu F, Qiu J, Fan Y, Zhang Q, Cheng B, Wu Y and Bai B: Apelin-13 attenuates ER stress-mediated neuronal apoptosis by activating $\mathrm{G} \alpha_{\mathrm{i}} / \mathrm{G} \alpha_{\mathrm{g}}-\mathrm{CK} 2$ signaling in ischemic stroke. Exp Neurol 302: 136-144, 2018

134. Manni S, Brancalion A, Tubi LQ, Colpo A, Pavan L, Cabrelle A, Ave E, Zaffino F, Di Maira G, Ruzzene M, et al: Protein kinase CK2 protects multiple myeloma cells from ER stress-induced apoptosis and from the cytotoxic effect of HSP90 inhibition through regulation of the unfolded protein response. Clin Cancer Res 18: 1888-1900, 2012.

135. Hessenauer A, Schneider CC, Götz C and Montenarh M: CK2 inhibition induces apoptosis via the ER stress response. Cell Signal 23: 145-151, 2011.

136. Fassier C, Tarrade A, Peris L, Courageot S, Mailly P, Dalard C, Delga S, Roblot N, Lefèvre J, Job D, et al: Microtubule-targeting drugs rescue axonal swellings in cortical neurons from spastin knockout mice. Dis Model Mech 6: 72-83, 2013.

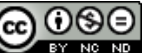

This work is licensed under a Creative Commons Attribution-NonCommercial-NoDerivatives 4.0 International (CC BY-NC-ND 4.0) License. 